\title{
Outcomes of a revised apprentice carpenter fall prevention training curriculum
}

\author{
Bradley Evanoff ${ }^{\mathrm{a}^{*}}$, Vicki Kaskutas ${ }^{\mathrm{a}}$, Ann Marie Dale ${ }^{\mathrm{a}}$, John Gaal ${ }^{\mathrm{b}}$, Mark Fuchs ${ }^{\mathrm{b}}$, and Hester Lipscomb ${ }^{\mathrm{c}}$ \\ ${ }^{a}$ Department of Medicine, Washington University, Campus Box 8005, 660 S. Euclid, St. Louis, MO 63110, USA \\ ${ }^{b}$ Carpenters' District Council of Greater St. Louis and Vicinity, Carpenters Joint Apprenticeship Program, 8300 \\ Valcour St. Louis, MO 63123, USA \\ ${ }^{c}$ Division of Occupational and Environmental Medicine, Duke University, Box 3834, Durham, N.C. 27710, USA
}

\begin{abstract}
Falls from heights are a leading cause of morbidity and mortality among construction workers, especially inexperienced workers and those performing residential construction. This research reports changes in fall prevention behaviors following revision of fall prevention training in a union-based carpenters' apprenticeship program. We used a comprehensive needs assessment to identify gaps in apprentice carpenters' preparation to work at heights, used these results to guide a schoolbased fall prevention curriculum to fill these gaps, and measured the effects of the revised curriculum on knowledge, beliefs, and fall prevention behaviors.
\end{abstract}

Keywords: construction safety, intervention study, fall prevention, safety training

\section{Introduction}

Falls from heights are a leading cause of morbidity and mortality among construction workers, especially inexperienced workers and those performing residential construction.

\section{Method}

We conducted a comprehensive needs assessment to identify gaps in an apprentice carpenters' fall prevention training and to guide curricular changes. Baseline apprentice questionnaires $(n=1,025)$, focus groups $(n=36)$, and worksite audits $(n=197)$ measured fall experience, and preferred learning methods, as well as domains of knowledge, risk perceptions, safety climate, and residential worksite behaviors. Baseline results were analyzed and compared to the existing fall prevention curriculum by a team of carpenter instructors and researchers. Training objectives and methods were designed to address gaps in the curriculum and integrate training methods preferred by this population.

The fall prevention curriculum for apprentice carpenters was revised based on results of the gap analysis, and included both changes in content, delivery, and timing [1]. Priorities of the revised training included safe ladder habits, truss setting, scaffold use, guarding floor openings, and using personal fall arrest systems. New apprentices were targeted to ensure that training was delivered prior to exposure at the workplace. The delivery of fall training was changed to incorporate principles of adult learning, with more active participation in the application of new learning. Hands-on experiences were provided through the use of a partially constructed house within the apprentice training school to practice fall protection behaviors in a realistic setting.

Following the revisions in apprentice training, apprentice questionnaires were re-administered on a yearly basis for four years, and residential worksites $(n=207)$ were re-audited one year after modifying the curriculum.

\footnotetext{
* $*$ Corresponding author. Tel. No.: $011+1+314+454+8638$, Email: bevanoff@dom.wustl.edu
} 
At each survey, we assessed the frequency of falls from height in the past year, with a fall defined as falling from one height to another. Among those who reported falling in the past year, we defined "serious" falls as those requiring medical care, prescription medications, light duty or restricted work, or lost work time beyond the day of the fall. Employer size was categorized by number of employees reported working for current contractor: small $(<=25)$, medium (26-75), and large ( $>75)$. Recent experience in residential construction was defined as working more than 3 months in residential construction in the past year. We used crew behavior and safety climate scales previously shown to be internally consistent [2].

To evaluate for changes in the survey domain scores following implementation of the modified training program, we ran linear regression models for each domain while adjusting for contractor characteristics and worker factors (contractor size, time in trade, proportion of apprentices in crew and reported recent residential experience). To determine whether training effects altered reported falls, we ran Poisson regression analyses to account for differences in number of hours worked by subject in the previous year, adjusting for time of survey, time in trade, safety climate, proportion of apprentices in the crew, and recent residential experience. In order to account for variation in work hours over time, we calculated a denominator of person time based on self-reported work hours.

The St. Louis Audit of Fall Risks was used to audit the fall safety of the worksites. This observational audit has been shown to be reliable and valid [3]. Audits were conducted at both time points by experienced construction workers, trained in the use of a multi-domain instrument designed for use in residential construction [3]. Safety compliance was computed for the audit and each domain by dividing the number of items that met safety criteria by the total number of items observed at the worksite or in the domain.

We evaluated the effects of the modified training by comparing worksite audits that were conducted before and after implementation of the program. We compared the overall audit score and the nine audit domains using paired t-tests. We ran linear regression analysis using the total audit score adjusting for the time of the audit (pre/post delivery of modified program), contractor size (small, medium, large) and proportion of apprentices at the worksite.
Analyses were conducted using the statistical software package SAS 9.2 (SAS Institute, Carey, NC) and SPSS Version 17.0 (SPSS Inc., Chicago IL).

\section{Results}

At baseline, we found that many apprentice carpenters perform work tasks at heights prior to training and that $16 \%$ report falling from height in the previous year [2].

The most common surface that apprentices fell from was a ladder (29\%), however ladder use was perceived as the task performed with the lowest risk level. Apprentices' knowledge of work safety standards was poor. Worksite audits showed many unsafe behaviors, including improperly erected ladders and unprotected openings and edges. Fall protection techniques were not commonly used at residential construction sites visited [4].

Following the training intervention, improvements were seen in the rate of falls, as well as in crew safety behaviors, safety climate, and safety knowledge.

There were fewer reported falls at the follow-up survey time points. Taking into account the amount of time worked in the preceding year, the rate of all falls per 10 person-years worked decreased at each survey $(1.85,1.50$, and 1.15 respectively) as did the rate of "serious" falls $(0.36,0.23$, and 0.17 respectively). Results from the Poisson regression analysis predicting the occurrence of falls showed that carpenters in the lowest quartile for safety climate scores were almost 2 times more likely to experience a fall compared to those in the safest group by safety climate score. Recent residential work and workers with less than one year of experience in the carpentry trade were found to be at a significantly greater risk of falling.

Crew behavior, safety climate, and knowledge scores all showed improvement after training. Results from the Poisson regression analysis predicting the occurrence of falls showed that carpenters in the lowest quartile for safety climate scores were almost 2 times more likely to experience a fall compared to those in the safest group by safety climate score. Recent residential work and workers with less than one year of experience in the carpentry trade were found to be at a significantly greater risk of falling.

Comparison of pre- and post-intervention worksite fall safety audits showed overall improvement in compliance with fall prevention behaviors (from 59\% to $75 \%, \mathrm{p}<.001$ ), with statistically meaningful im- 
provement seen in six of the nine audit domains. Linear regression results showed that these improvements in compliance persisted after adjustment for contractor size and the percentage of the workforce who were apprentices at each worksite (postintervention audit scores were $14.9 \%$ higher, $95 \% \mathrm{CI}$ : $10.0 \%-19.9 \%$ ). Small and medium sized contractors had lower overall fall safety audit scores compared to large contractors $(16.8 \%$ (95\% CI: $8.4 \%-25.1 \%)$ and $10.0 \%$ (95\% CI: 3.8\% - 16.6\%), respectively).

\section{Discussion}

We implemented a multi-faceted fall prevention training intervention guided by data from a comprehensive needs assessment, and designed to meet apprentices' learning needs and preferences. This curriculum intervention was followed by measureable improvements in reported falls, fall prevention knowledge, and safety climate. We also saw improvements in both self-reported safety behaviors and in observed fall prevention behaviors among residential carpentry work crews.

There are important limitations to this study, as the study design - a pre- post-intervention design without a concurrent control group - limits the strength of the conclusions that may be drawn from our findings. Other factors may account for the improvements in fall prevention behaviors we observed, including a change in the makeup of the workforce or nature of the work due to the downturn in new home construction that occurred during our study period. The observed improvements may also have occurred as a consequence of changes in the safety training activities and safety culture of construction contractors who employed our study group. Anecdotal evidence suggests that several contractors who were involved in our study made changes in safety policies and training during the study period. This can be viewed as a confounder in the analysis of our apprentice training school-based intervention; it can also be viewed as a result of the intervention. Also, our study was designed to measure changes in knowledge, attitudes, and behaviors related to the training intervention, and was not powered to detect changes in mortality or morbidity related to falls.

Our study had several strengths, including the sampling of a large group of apprentices at different stages of training, and the high participation rates in surveys (98\%) and worksite audits (96\%). Importantly, our study measured observed safety behaviors at almost 400 worksites in addition to self-reported behaviors. We benefited from a close collaboration between the apprenticeship program, the carpenters' union, and the homebuilders. The intensity of the evaluation was made possible by this organized environment. Though our evaluation would be very difficult to complete outside of a union-based apprenticeship, elements of our training program could readily be adapted for use in other groups of union and nonunion workers. The worksite audit could also be used by other researchers and construction professionals.

Our intervention affected the training that apprentice carpenters received in the school-based portion of their apprenticeship, but not the training and mentoring they received on the job. Previous work by our team [2,5] suggests that inexperienced carpenters would benefit from improved mentorship from foremen and journeymen on their work crews. Future work is needed to improve the training and mentoring received by early-stage workers, who are at significantly higher risk of injury than more experienced carpenters.

\section{Acknowledgements}

We would like to thank our collaborators, including the Carpenters' District Council of Greater St. Louis and Vicinity, St. Louis Carpenters' Joint Apprenticeship Program, the Home Builders' Association of St. Louis and Eastern Missouri, and the residential contracting companies and carpentry professionals who participated in this research.

\section{References}

[1] V. Kaskutas, A.M. Dale, H. Lipscomb, J. Gaal, M. Fuchs, B. Evanoff, and Carpenters' Joint Apprenticeship Program Instructor Team, Changes in fall prevention training for apprentice carpenters based on a comprehensive needs assessment, J Safety Res (2010), 221-7.

[2] V. Kaskutas, A.M. Dale, H. Lipscomb, J. Gaal, M. Fuchs, and B. Evanoff, Fall prevention among apprentice carpenters, Scand J Work Environ Health (2010), 258-65.

[3] V.K. Kaskutas, A.M. Dale, H.J. Lipscomb, and B.A. Evanoff, Development of the St. Louis audit of fall risks at residential construction sites, Int J Occup Environ Health (2008), 243-9.

[4] V. Kaskutas, A.M. Dale, J. Nolan, D. Patterson, H.J. Lipscomb, and B. Evanoff. Fall hazard control observed on residential construction sites, Am J Ind Med (2009) 491

[5] H.J. Lipscomb, A.M. Dale, V. Kaskutas, R. ShermanVoellinger, and B. Evanoff B, Challenges in residential fall prevention: insight from apprentice carpenters, Am J Ind Med (2008), 60-8. 